\title{
Design of three Ring Resonator SOI Structure for Cholesterol Detection in Blood
}

\author{
Sai Preethi Jatta, Sharath K Prabhu, Vismaya K R and Shwetha M \\ Sai Vidya Institute of Technology, Department of ECE, Bengaluru, India - 560064
}

\begin{abstract}
In this paper work, a three ring resonator SOI structure with two bus waveguides and three ring waveguides is implemented, which is made to operate in the near infrared region. The detection of the presence of cholesterol having a RI of 1.53 in blood at $1550 \mathrm{~nm}$ wavelength has been analyzed. There has been a significant shift of the resonance peak by $100 \mathrm{~nm}$ in the presence of cholesterol in human blood as an analyte which can be used as a detection mechanism for hazardous levels.
\end{abstract}

\section{INTRODUCTION}

The sensing of bio-molecules like proteins, cholesterol, carbohydrates and DNA is gaining a great interest in the areas such as medical diagnostics. The Rapid advances in bio-medical field have led to the expansion of various instruments for detection and prevention applications. In the past few years, optical ring resonators have emerged as a popular sensing technology for highly sensitive detection of analytes in fluids. In this structure, we have designed a three ring resonator and performed analysis of its spectral properties to obtain a sensor with high quality factor, free spectral range (FSR), finesse and full width half maximum (FWHM) [1].

Light confined within a propagating waveguide undergoes evanescent field sensing. Even if the light is allowed to propagate through the guiding region, a part of it extends outside and interacts with the surrounding medium. This type of sensor exhibits very high sensitivity and is also helpful in mass production. Silicon on insulator (SOI) biosensing technology is used because it combines all the advantages which includes deep UV lithography, mass fabrication and integration, developing real lab-on-chip devices and also for a various bio sensing applications [1].

Ring resonator, having been one of the most promising biological sensors, it measures the target molecule by observing the deviations in light behavior. After sensing the existence of the particle, the effective refractive index of the medium changes, which results in the frequency shift. Refractive index of 3.47 as silicon is highly used in nano fabrication and is also one of the best suited material for highly sensitive integrated optical devices [2].

Any variations in the refractive index is observed through the ring resonator's optical properties like quality (Q) factor, finesse, etc. The Q-factor is calculated based on the resonant

Vol. 3 (1), July 2019, www.ijirase.com peaks and is found to be maximum. Sensitivity is also taken into consideration and is maintained accordingly. It is observed for a three ring resonator in between two bus waveguides which are configured in a parallel manner. ${ }^{[3]}$

Optical filters are generalized interferometers which splits the incoming signal into many paths, in an essentially wavelength independent manner, delayed and recombined. The splitting and recombining ratios as well as delays are varied to change the frequency response. A glass silica waveguide is typically used to find the widest FSR [4-6]

In this paper work, a three-ring resonator is connected in parallel and is placed in between two bus waveguides. Light source with a wavelength around near infrared is used to see the shift in power when a liquid is placed inside the ring. Here, we have used cholesterol as an analyte. It could be considered as an extension of one of the paper works in which liquid crystal is used. Finding out one of the biomolecule's response and finding its Q-factor is shown throughout [7-8].

\section{THEORY}

Cholesterol $\left(\mathrm{C}_{27} \mathrm{H}_{46} \mathrm{O}\right)$ is an organic compound of alcohol found in most of the body tissues. It has a molecular weight of $386.64 \mathrm{~g} / \mathrm{mol}$. It is also present as a waxy substance found in the blood. Predominantly it is produced in the liver for certain metabolism in the body. It is also found in foods such as red meat, high fat cheese, butter and eggs. It is essential for maintaining good health and only becomes a problem when the level in your blood is too high. High levels of cholesterol is a major risk factor in the development of coronary heart disease. However, proper diagnosis and detection at early stages can reduce the risks of potential threats which can be lethal enough to cause deaths.

Cholesterol has a Refractive Index of 1.53. Exploiting this property of the fatty substance in human blood, we have analyzed a detection process using the ring resonators. A given ring resonator structure has a particular frequency of resonance for which the phenomenon of evanescence takes place between the waveguide structures. In this paper, a light of wavelength ranging from $1.5 \mu \mathrm{m}$ to $1.6 \mu \mathrm{m}$ is used as a source of light for its detection. A blood sample with high cholesterol index is subjected to study using the property of resonance shift of a ring resonator. When a ring resonator with a characteristic resonant wavelength is employed with an analyte of cholesterol contained blood within the rings of the ring resonator structure, there is a consequential spectral 
shift in the resonant wavelength. The magnitude of the spectral shift can determine the amount of cholesterol levels in the blood which can substantiate the vulnerability of health risks. This process can be deployed for medical diagnosis to estimate the cholesterol levels.

\section{DESIGN}

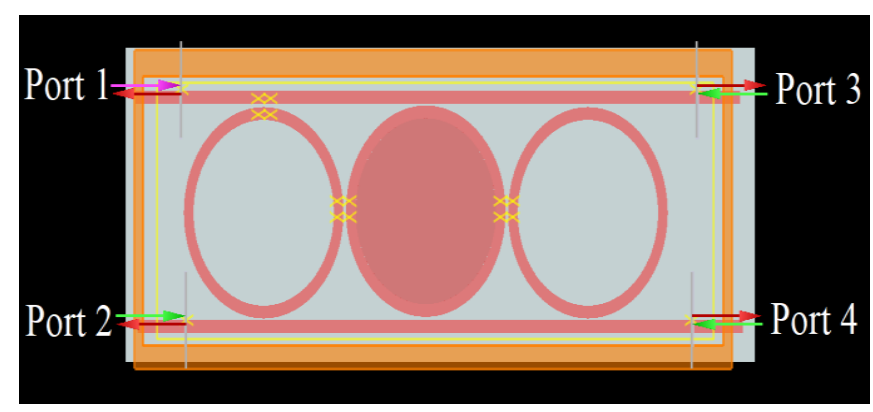

Figure 1: Three ring resonator structure

This ring resonator structure with a width of $250 \mathrm{~nm}$ and a height of $400 \mathrm{~nm}$ is implemented by using Lumerical FDTD software. Silicon is used as a material for the ring and bus waveguides because of its high refractive index. $\mathrm{SiO}_{2}$ is used as a substrate base over which the waveguides are placed. The three identical ring waveguides have been designed with a radius of $3.3 \mu \mathrm{m}$ and are arranged in a parallel manner. The structure is analysed by placing four port monitors at each end of the two bus waveguides respectively. The light is injected at Port1 on the upper bus waveguide. It evanescently couples with the ring waveguides which are placed in parallel with them. There after itcouples with the lower bus waveguide and finally the light will exit through the Port 3 which is considered as the output port. The dropped wavelength of the incident light source can be observed at port 2 . The ring resonator structure is depicted in figure 1 .

The Q-factor is calculated by placing 3 monitors, two of which are placed between the rings and one at the intersection of ring waveguide and bus waveguide.

\section{RESULTS AND DISCUSSION}

The simulation results are obtained using the Lumerical FDTD software. The rings are placed in between the bus waveguide and the coupling of light is observed in the $\mathrm{E}$ and $\mathrm{H}$ fields.

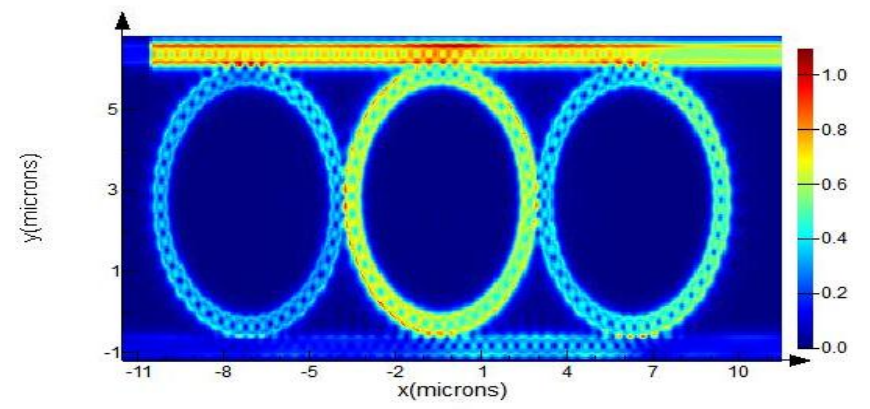

Figure 2: Full profile of coupling at a wavelength of $1.6 \mu \mathrm{m}$

Cholesterol is chosen as the analyte that will be placed inside the ring to study as shown in figure. 1. In Figure 2 the coupling of light at mid infrared wavelength in electric field is observed. The light is made to pass from the Port.1 through the upper bus waveguide. It couples with the first ring and then into the other two rings and finally into the lower bus waveguide. The light finally exits through thePort.3.

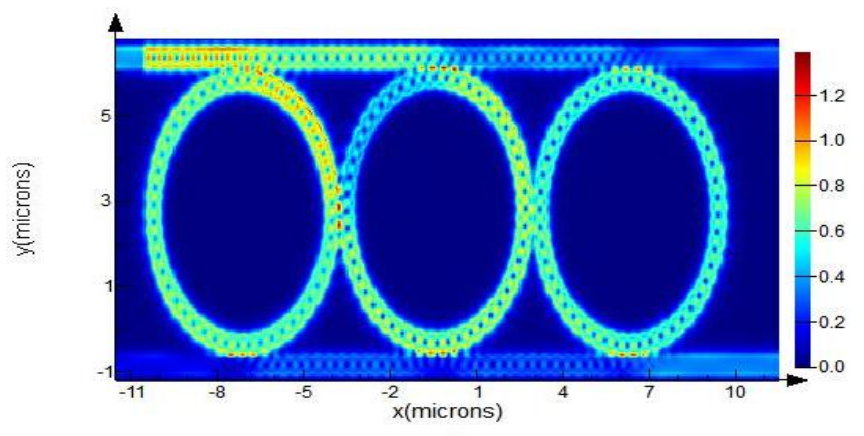

Figure 3: Coupling of light at $1.54839 \mu \mathrm{m}$

The figure. 3 and figure. 4 shows the evanescent coupling of light at $1.54839 \mu \mathrm{m}$ and $1.57377 \mu \mathrm{m}$ wavelength respectively. The intensity of light is maximum at the Port 1 at the source. After multiple round trips of constructive interference it exits through Port2, Port3 and Port4.

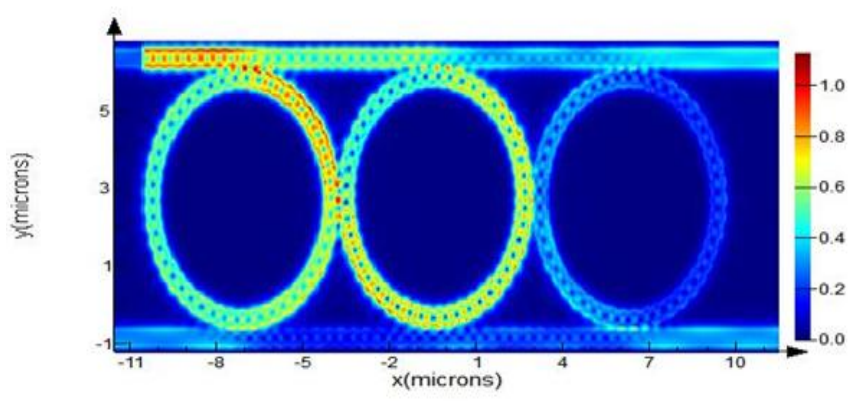

Figure 4: Coupling of light at $1.57377 \mu \mathrm{m}$

The input power at Port1 will be high. After multiple couplings through the three rings and the bus waveguide the light entering the Port3 is monitored. 
Figure.5(a) and Figure.5(b) depict the transmittance and power at the input port respectively. This gives us the information about the power and transmittance before the light interacts with the analyte. The resonance peak is observed at $0.3 \mu \mathrm{m}$ approximately.

The coupling through the ring waveguides is given by the troughs in the transmission plot. The free spectral range, is given by the distance between two adjacent peaks, it is one of the spectral properties of the optical devices. It is convenient to detect the analyte or bio-molecule as long as the light is confined in the ring waveguides. The scattering of light and the transmission play an important role in identifying the bio-molecule.

The spectral properties such as Q-factor, FSR, wavelength and power variations must be considered for improvising the sensitivity in biosensing applications.

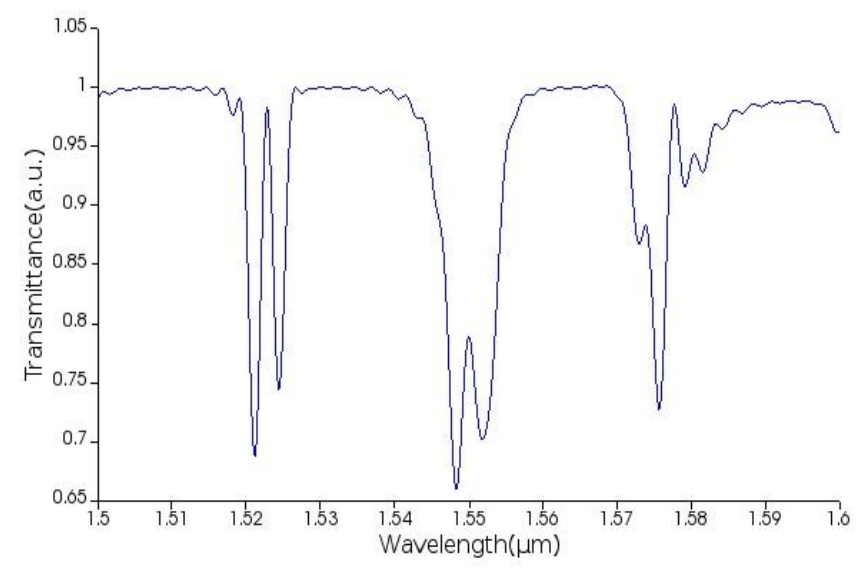

Figure 5(a): Transmittance at port 1

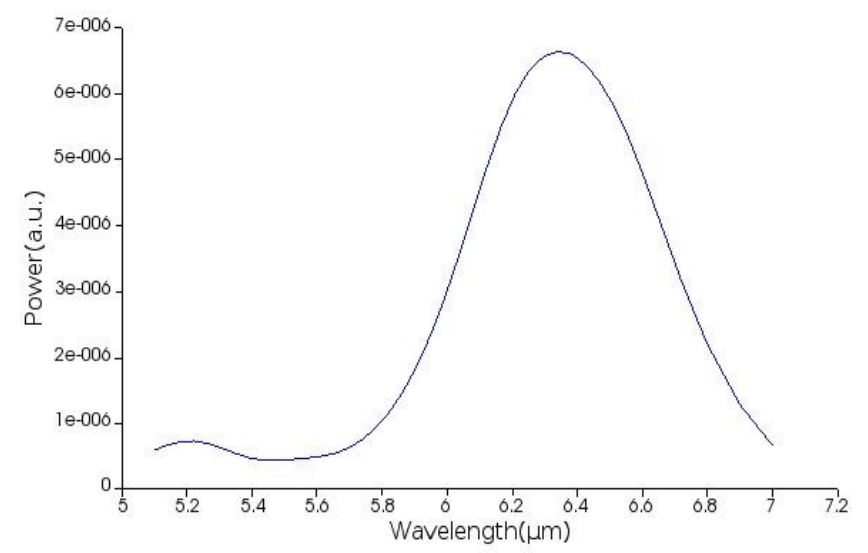

Figure 5(b): Power at port 1

Figure.6(a) and Figure.6(b) depict the transmittance and power at the port3 respectively. This gives us the information about the power and transmittance after the light successfully interacts with the analyte. It can be observed that there is a shift in the resonance peak observed at $0.4 \mu \mathrm{m}$ by $100 \mathrm{~nm}$ for the wavelength ranging from $1.5 \mu \mathrm{m}$ to $1.6 \mu \mathrm{m}$ which is the required for the simulation of the designed structure.

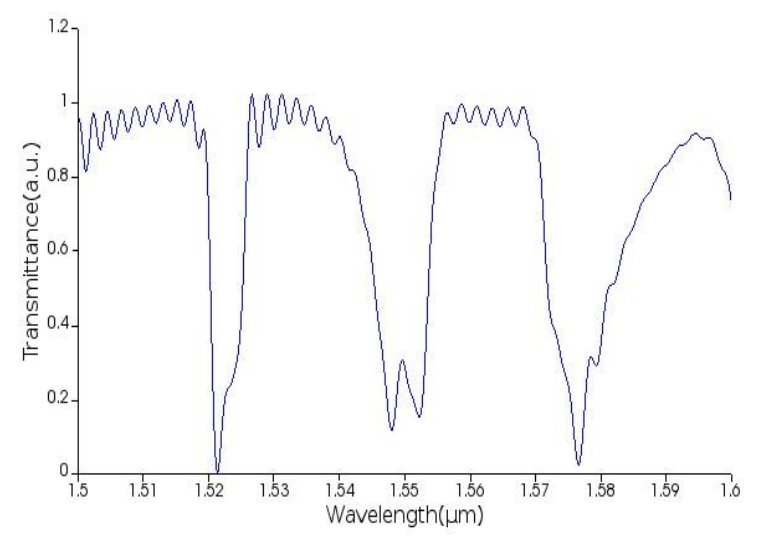

Figure 6(a): Transmittance at port 3

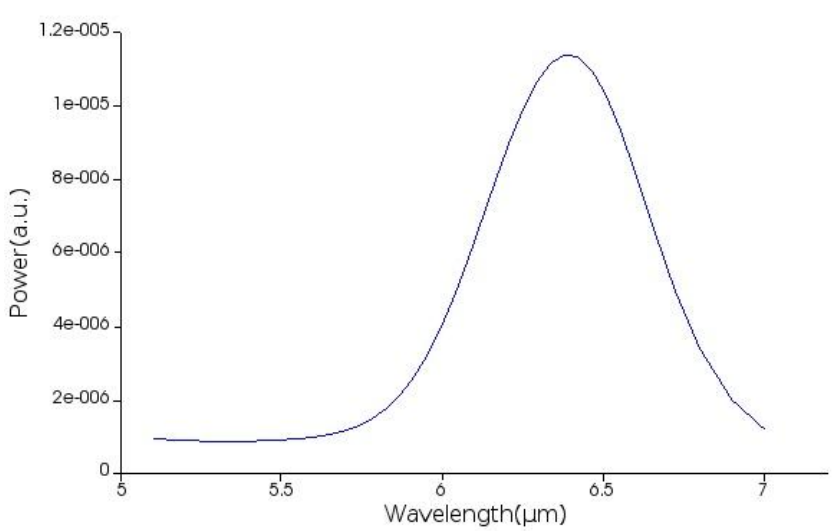

Figure 6(b): Power at port 3

The table 1 shows the Q-factor for different wavelengths. In the table the $\mathrm{Q}$ factor has been observed at various wavelengths, where the maximum Q-factor is observed as 5260 for the three coupled ring resonator.

TABLE I

Q-FACTOR AT DIFFERENT WAVELENGTH.

\begin{tabular}{|l|l|}
\hline $\begin{array}{l}\text { Frequency } \\
\text { (THz)/Wavelength(nm) }\end{array}$ & Q-factor \\
\hline $200.322 \mathrm{THz} / 1496.56 \mathrm{~nm}$ & $1540.84+/-172.674$ \\
\hline $196.719 \mathrm{THz} / 1523.96 \mathrm{~nm}$ & $2670.17+/-1230.66$ \\
\hline $207.205 \mathrm{THz} / 1446.84 \mathrm{~nm}$ & $5259.62+/-2797.72$ \\
\hline $197.105 \mathrm{THz} / 1520.98 \mathrm{~nm}$ & $2067.35+/-1665.99$ \\
\hline $200.322 \mathrm{THz} / 1496.56 \mathrm{~nm}$ & $1180.13+/-130.383$ \\
\hline $197.105 \mathrm{THz} / 1520.98 \mathrm{~nm}$ & $2201.62+/-8193.69$ \\
\hline
\end{tabular}




\section{CONCLUSION}

A triple ring resonator with evanescence field coupling is chosen and two bus waveguides are placed in order to send and receive and also to see the dropped transmission rate. With cholesterol as one of the bio molecules with a refractive index of 1.53, transmittance and power is monitored. A ring resonator is designed to find the cholesterol level and is useful in bio-sensing. An SOI based material is used to make the fabrication easier and also to maintain sensitivity. Here, we have calculated the Q-factor with respect to the resonant peaks. The Q- factor is observed to be 5620 , which is maximum for the three ring resonator when compared with two and single ring resonator.

\section{FUTURE WORK}

This ring resonator model can be further improvised by adding two more analytes in the other two rings and the results can be simultaneously detected for all the three analytes at a time for a particular wavelength range. Responses could be varied because of the variation in the refractive indices of the analytes.

\section{ACKNOWLEDGMENT}

This work is supported by Vision Group on Science and Technology, Department of IT, BT \& ST, Government of Karnataka, India. Grant number: VGST - CISEE GRD - 466

\section{REFERENCES}

[1] Mario Iodice1, "Label-free biosensing by means of optical micro-ring resonator," Institute for Microelectronics and Microsystems - National Council of Research, Dept. of Naples, Via P. Castelli no 111, 80131 Naples, Italy. -K. Chen, Linear Networks and Systems, Proc. of SPIE Vol. 7356 735603-7.

[2] AzadehKiamSarkaleh, BabakVosoughiLahijani, HamidrezaSabekari, "Optical ring resonators: A platform for Biological Sensing Applications," Journal of Medical signals and sensors, July-Sept 2017.

[3] Shwetha M., Raksha V, Narayan K., "Analysis of ring resonator structure with quality factor enhancement," Proc. SPIE 11031, Integrated Optics: Design, Devices, Systems, and Applications V, 1103116 (26 April 2019); doi: 10.1117/12.2520946.

[4] SabitabrataDey, Sanjoy Mandal, "Enhancement of Free Spectral Range in Optical Triple Ring Resonator: A Vernier Principle approach", 978-1-4577-0697-4, 2012 published, IEEE.

[5] Shwetha M and K. Narayan, "A Ring Resonator with Liquid Crystal for Biosensing Application", IEEE Xplore, DOI: 10.1109/WRAP.2017.8468552.

[6] BhVijayaaditya, TalabatullaSrinivas, "Analysis of mutually coupled three ring resonator," IISc.

[7] SabitaBrataDey, S Mandal, Performance of a wide range FSR optical double ring resonator, 2009 International Conference on Emerging Trends in Electronic and Photonic Devices \& Systems (ELECTRO-2009).

[8] Senichi Suzuki, Kazuhiro Oda, Yoshinori Hibino, "Integrated Optical double ring resonators with a wide Free Spectral Range of 100GHz" Journal of Light Wave Technology, Vol No:13, August 1995.

[9] Dr Dominik G Rabus, "Integrated Ring Resonators, The Compendium, Springer Publications, 2007. 